\title{
Association Between Enrollment in an Enhanced Recovery Program for Colorectal Cancer Surgery and Long-Term Recurrence and Survival
}

Andrés Zorrilla Vaca ( $\square$ andres.zorrilla@correounivalle.edu.co) University of Texas MD Anderson Cancer Center https://orcid.org/0000-0001-8140-8486 Javier Ripolles-Melchor

Hospital Universitario Infanta Leonor: Hospital Infanta Leonor

Ane Abad-Motos

Hospital Universitario Infanta Leonor: Hospital Infanta Leonor

Inés Rubiera Mingu

Hospital Universitario Infanta Leonor: Hospital Infanta Leonor

Nekane Moreno-Jurado

Hospital Universitario Infanta Leonor: Hospital Infanta Leonor

Fátima Martínez-Durán

Hospital Universitario Infanta Leonor: Hospital Infanta Leonor

Isabel Pérez-Martínez

Hospital Universitario Infanta Leonor: Hospital Infanta Leonor

Alfredo Abad-Gurumeta

Hospital Universitario Infanta Leonor: Hospital Infanta Leonor

María L. FuenMayor Varela

Hospital Universitario Infanta Leonor: Hospital Infanta Leonor

Gabriel E. Mena

UTMDACC: The University of Texas MD Anderson Cancer Center

Michael C. Grant

Johns Hopkins Medical Institutions: Johns Hopkins Medicine

\section{Research Article}

Keywords: Enhanced Recovery After Surgery, Colorectal Surgery, Anesthesiology, Perioperative Medicine

Posted Date: September 3rd, 2021

DOI: https://doi.org/10.21203/rs.3.rs-870094/v1 
License: (c) (i) This work is licensed under a Creative Commons Attribution 4.0 International License. Read Full License 


\section{Abstract}

Introduction

Enhanced Recovery After Surgery (ERAS) programs have been shown to minimize the surgical inflammatory response in colorectal cancer, leading to early patient recovery and better postoperative outcomes. Our objective was to determine the association between an ERAS program for colorectal cancer surgery and oncologic recurrence and survival.

Methods

A before-after intervention study was designed including patients who underwent colorectal cancer surgery between November 2010 and March 2016. During the study period the institutional criteria for adjuvant therapy remained unchanged and all patients were followed up for 5 years. Cox hazard regression analysis was performed per cumulative year of follow up to evaluate the association between ERAS program exposure and overall survival, cancer-related mortality, and oncologic recurrence. Subgroup analysis was performed by cancer stage (low [I/II] vs advanced [III/IV]).

Results

In total, 612 patients were included, of which 321 were pre-ERAS and 291 ERAS. Our overall median compliance rate with ERAS interventions was 90\% (IQR 85\%-95\%). Overall survival rates were higher in the ERAS group within the first 2-years after surgery ( $89.2 \%$ vs $83.2 \%, P=0.04)$, but there was no difference at 5 -year follow up $(73.3 \%$ vs $72.5 \%, P=0.82)$. Subgroup analysis revealed the ERAS enrollment was associated with a significantly lower risk in 5-year oncologic recurrence (aHR $0.55,95 \% \mathrm{Cl} 0.33-0.94$, $\mathrm{P}=0.03$ ) and higher 4-year survival (aHR $0.59,95 \% \mathrm{Cl} 0.37-0.93, \mathrm{P}=0.02)$ among patients with advanced cancer stage compared to pre-ERAS counterparts.

Conclusions

Patients with advanced colorectal cancer were less likely to suffer oncologic recurrence when managed during the ERAS period. Additional prospective trials are necessary to determine causation and identify best practice principles associated with long-term recurrence and survival.

\section{Key Points}

- It is unknown whether Enhanced Recovery Programs (ERPs) are associated with cancer recurrence and survival.

- This retrospective study revealed improved survival within the first two years after colorectal cancer surgery among those who participated in an ERAS program.

- Oncological recurrence rates were significantly lower among patients with advanced colorectal cancer (III/IV) who received ERPs. 


\section{Introduction}

Cancer recurrence increases patient morbidity and represents a significant economic burden for health care systems. ${ }^{1}$ Although significant advancements have been made in surgical care and associated therapy, recurrence rates are still high depending on the quality of care provided during cancer surgery. ${ }^{2}$ Literature has shown that multidisciplinary perioperative care models facilitate early recovery and hasten the return to indicated medical oncologic therapy, ${ }^{3}$ which may reduce cancer recurrence following surgery and improve long-term survival. ${ }^{4}$ Nevertheless, there is a lack of knowledge regarding the association between specific perioperative interventions (e.g., anesthetic type, pain regimen, nutrition, rehabilitation) and long-term oncologic outcomes. ${ }^{5}$

Enhanced Recovery After Surgery (ERAS) protocols involve the bundled application of evidence-based perioperative care interventions which are primarily aimed at hastening patient recovery and reducing surgical stress response. ${ }^{6}$ In recent years, the ERAS ${ }^{\circledR}$ Society has compiled a set of guidelines across multiple surgical specialties, ${ }^{7}$ all of which support the safety and efficacy of this multidisciplinary model as a standard of care to achieve better patient satisfaction, superior pain control, and lower postoperative morbidity. ${ }^{8}$ As new evidence accumulates in favor of ERAS, more research is now targeting longer term outcomes, such as survival in oncologic patients. ${ }^{9}$ Unfortunately, the evidence regarding management associated with an ERAS program and cancer recurrence is still inconclusive, with a handful of studies yielding inconsistent results about the impact on survival. ${ }^{10-13}$ Our group aimed to determine the association between an ERAS program for colorectal surgery and long-term cancer recurrence and survival.

\section{Methods}

\section{Design}

This is a retrospective, cohort study conducted in a single institution, community-based academic hospital with the same group of anesthesiologist and surgeons during the study period. The protocol for this study was approved by our institutional review board and all data was de-identified to protect confidentiality. Informed consent was waived due to the retrospective nature of this study. Two groups of patients were identified based on the period of ERAS initiation in our institution (March 2013). Patients who received conventional perioperative care underwent surgery from November 2010 through January 2013 (Pre-ERAS group). Patients who received perioperative care according to the ERAS protocol underwent surgery from March 2013 through March 2016 (ERAS group).

\section{Inclusion \& Exclusion Criteria}

This study included adult patients undergoing elective colorectal surgery. Emergent procedures were excluded, along with any patient who was unable or unwilling to participate in the ERAS program. 


\section{Variables}

Demographics and clinical variables were extracted from electronic medical records. Colorectal cancer was classified based on the American Joint Committee on Cancer (AJCC) / Dukes stages, ${ }^{14}$ as follows: Stage I (T1, T2, N0, M0), Stages II (T3, T4, N0, M0), Stage III (any T, N1, N2, M1), Stage IV (distant metastasis).

\section{Outcomes}

Our primary outcome was overall 5-year survival, which was defined as the living or deceased status after surgical treatment. Time-to-death was also recorded for the purpose of the analysis. This was assessed through hospital and primary care medical records. It was classified into 3 categories, including postoperative causes, defined as death secondary to postoperative complications occurring in the first 30 days after surgery; oncologic causes, defined as secondary to tumor progression despite planned curative surgical treatment; and other causes, defined as those not due to tumor progression or postoperative, not related to disease (i.e., accident, or other illness).

Oncologic recurrence was defined as the identification of a tumor mass consistent with the primary cancer in any part of the body, after surgery or any treatment modality with curative intent. This was typically performed through an active surveillance based on the cancer stage ( $I$ - annual colonoscopy, II/III/IV - clinical review at least every 6 months during the first 3 years and annually until the fifth year with carcinoembryonic antigen, chest X-ray and abdominal ultrasound, annual colonoscopy were performed). The time to first recurrence was also recorded.

\section{Statistical Analysis}

An initial exploratory analysis was performed using descriptive statistics. Univariate analysis compared demographics and clinical variables between pre-ERAS and ERAS periods. Kaplan Meier curves were plotted along with log-rank $P$ values in order to identify potential differences in time to mortality events or recurrence events between both periods. Cox hazard regression analysis was performed at each year of follow up to evaluate the association between ERAS program enrollment and overall survival, cancerrelated mortality, and oncologic recurrence. Certain clinically relevant confounders (i.e., age, ASA, cancer stage, and comorbidities) were included in the multivariable survival analysis. Hazard ratios (HR) were reported along with their corresponding $95 \%$ confidence intervals $(\mathrm{Cl})$. Survival rates and oncologic recurrence were also evaluated in subgroup analysis based on cancer stage (low [I/II] vs advanced [III/IV]). $\mathrm{P}<0.05$ was considered significant for all analyses. The initial descriptive analysis was done in Stata 14.0 (StataCorp, College Station, TX), and the survival analysis was conducted in the R Stats Package (Statistical Computing, Vienna, Austria).

\section{Results}

\section{Patient Characteristics}


A total of 612 patients were included, 321 of which were pre-ERAS and 291 ERAS. Our overall median compliance rate with the ERAS protocol was 90\% (IQR 85\%-95\%). There was a greater proportion of female patients in the ERAS period ( $42 \%$ vs $32 \%, P=0.03)$. The remainder of baseline demographic and clinical characteristics were comparable between periods (Table 1). Patients enrolled in the ERAS program had shorter surgical times (119 vs. 140 minutes, $P<0.01$ ), received more total intravenous anesthetics (TIVA; 24.7 vs. 13.9\%, $\mathrm{P}<0.01)$, fewer epidurals (10.2 vs. $21.0 \%, \mathrm{P}<0.01)$ and less perioperative fluid (1970 vs. $2183 \mathrm{~mL}, \mathrm{P}<0.01$ ) compared to pre-ERAS counterparts.

\section{Survival Analysis}

There was no difference in overall 5-year survival between groups (71.5\% ERAS vs. 73.3\% pre-ERAS, $\mathrm{P}=0.31)$. Survival rates were greater at year 1 (94.8\% ERAS vs $89.8 \%$ pre-ERAS, $\mathrm{P}=0.02)$ and year $2(89.2 \%$ ERAS vs 83.2\% pre-ERAS, $\mathrm{P}=0.04$ ) in the ERAS group, but no difference was detected thereafter. Kaplan Meier curves of each year epoch are illustrated in Figure 1. There was no difference in the proportion of cancer-related deaths (15\% ERAS vs. $17 \%$ in pre-ERAS, $P=0.35$ ) and survival time was not statistically different (2.3 years [1.7-3.3] ERAS vs. 2.1 years [1.1-3.8] pre-ERAS, $P=0.61)$.

Subgroup analysis by cancer stage demonstrated an effect modification. Although low cancer stage did not reveal an association with survival at 5 years of follow up (77\% ERAS vs. 83\% pre-ERAS, P=0.13), patients with advanced cancer stage who were enrolled in ERAS experienced a statistically significantly higher 5-year overall survival (67 vs. $55 \%, \mathrm{P}=0.05)$ compared to those who received conventional care. $A$ similar association was confirmed among patients with advanced cancer stage via multivariable analysis in year 1 (aHR $0.24,95 \% \mathrm{Cl} 0.09-0.62, \mathrm{P}=0.003)$ through year $4(\mathrm{aHR} 0.59,95 \% \mathrm{Cl} 0.37-0.93, \mathrm{P}=0.02)$ of the study follow up period.

Disease-free 5-year survival did not differ between groups (66\% ERAS vs. 60\% pre-ERAS, P=0.14), but it was significantly higher in the ERAS group compared to conventional care among patients with advanced cancer stage (58\% ERAS vs. 39\% pre-ERAS, $\mathrm{P}<0.01)$. While there a was no difference in disease-free 5year survival between groups among low cancer stage (aHR 1.11, 95\% Cl 0.74-1.67, P=0.59), advanced cancer stage patients who were enrolled in ERAS had better disease-free 5-year survival $(\mathrm{aHR} 0.53,95 \% \mathrm{Cl}$ 0.36-0.77, $\mathrm{P}<0.01$ ) compared to conventional care counterparts.

\section{Oncologic Recurrence}

Overall recurrence rates (17\% ERAS vs. $21 \%$ pre-ERAS, $\mathrm{P}=0.31)$ as well as time to recurrence (1.56 years [0.9-2.0] ERAS vs. 1.15 years [0.5-2.4] pre-ERAS, $P=0.15)$ was similar. Subgroup analysis revealed that patients with advanced cancer stage experienced lower oncologic recurrence (22\% in ERAS vs. $32 \%$ in preERAS, $P=0.05)$, a result that was confirmed after adjusting for potential confounders $(\mathrm{aHR} 0.55,95 \% \mathrm{Cl}$ $0.33-0.94, P=0.03)$. There was no difference in recurrence rates among patients with low cancer stage (aHR 0.96, 95\% Cl 0.53-1.72, $\mathrm{P}=0.89$ ).

\section{Discussion}


The results of this study found several important associations between care administered through an ERAS program and survival after colorectal surgery. First, we did not detect a difference in overall survival, disease free survival or oncologic recurrence at 5 years. However, subgroup analysis revealed an association between ERAS program enrollment and survival, disease free survival and oncologic recurrence among patients with advanced cancer stage compared to those who received conventional care. These associations remained statistically significant after adjustment for a number of potential confounders. These findings suggest that care administered through an ERAS program may impact not only immediate postoperative rates of recovery as demonstrated in previous studies, but potentially play a role in longer term outcome after curative surgical resection.

Our findings align with previous studies that have showed improved survival rates associated with ERAS. Lohsiriwat et al. conducted a similar cohort study and showed ERAS was associated with improved survival among a subgroup of patients with stage III cancer. ${ }^{13}$ Additionally, high compliance $(>70 \%)$ with an ERAS program has been correlated with better 5-year survival rates in advanced stages of cancer, ${ }^{12,13}$ but not at 3-years of study follow up. ${ }^{11}$ Quiram et al. ${ }^{10}$ found a statistically significant association between ERAS and overall survival, but no relationship was detected for disease-free survival. As shown, our study demonstrated a significant improvement in both overall and disease-free survival rates among patients with advanced initial cancer stage.

There are several reasons to suspect that interventions included within an ERAS program may positively influence survival rates after surgery. In a recent trial, prehabilitation was associated with improved 5-year disease-free survival in patients undergoing colorectal surgery. ${ }^{15}$ Minimally invasive surgical technique, evaluated in a recent meta-analysis, was shown to yield better survival compared to an open approach following colorectal cancer resection. ${ }^{16}$ Fluid therapy optimization may also be contributing to better survival rates within ERAS according to the results presented by Asklid et al, ${ }^{17}$ who demonstrated that restrictive perioperative fluid therapy ( $\leq 3000 \mathrm{~mL}$ on the day of surgery) is associated with a $55 \%$ increase in 5-year survival. A number of other observational trials have identified an association between certain anesthetics, analgesics (i.e., neuraxial) and reduced opioid administration and subsequent cancer recurrence and rates of survival. ${ }^{18}$ According to the PACO-RAS trial, peridural analgesia, as part of a multimodal regimen, may be associated with improved survival, ${ }^{19}$ although similar attempts to reproduce those results have yielded conflicting results. ${ }^{20}$ It is feasible that incremental gains provided by several interventions shown to reduce inflammation and prevent immunosuppression associated with surgical insult, the net effect potentially being long term reductions in cancer recurrence and improved survival.

ERAS programs are associated with fewer postoperative complications (e.g., ileus, anastomotic leak, surgical site infections), which may underpin short-term benefits. Our program previously demonstrated that interventions were associated with reduced moderate and severe complications compared to conventional care. ${ }^{21}$ This may have influenced survival within the first two years. However, our analysis also revealed an association between ERAS and lower cancer-related deaths and oncologic recurrence. As theorized previously, this can be explained either through reduced surgical insult and associated 
inflammation. ${ }^{22}$ For instance, Cabellos-Olivares et al noted a reduced systemic inflammatory response as indicated by C-reactive protein (CRP) after implementing ERAS in colorectal surgery. ${ }^{23}$ Venara et al observed less expression of arachidonic acid metabolism in patients managed with ERAS protocols, particularly a reduction in microsomal prostaglandin $E$ synthase and hematopoietic prostaglandin $D$ synthase. ${ }^{24}$ Jaloun et al identified lower neutrophil/lymphocyte ratios in patients treated under ERAS protocols compared to conventional care. ${ }^{25}$ An alternative therapy altogether may be that faster recovery leads to the hastened ability to undergo subsequent intended oncologic therapy, which may be particularly true for patients with advanced cancer stage (III/IV), who experienced the greatest improvement in survival with ERAS implementation.

This study has several important limitations, including its retrospective design, which obviously precludes establishing causality. Though we attempt to address relevant confounders, we cannot exclude the potential for unmeasured or uncaptured variables that may impact the analysis. Unfortunately, data regarding metastatic disease and relevant neoadjuvant therapy is not available, which prevents us from evaluating for association between subsequent oncological therapy and overall rates of recurrence and survival. However, the selection criteria for adjuvant and neoadjuvant therapy did not differ between study periods and with the exception of gender, the patients had comparable demographic and clinical characteristics.

\section{Conclusion}

Although enrollment was not associated with a difference in survival at 5-years after surgery, patients who received perioperative care within an ERAS program with advanced colorectal cancer did have improved survival and lower likelihood of oncologic recurrence compared to conventional care. These findings should be considered hypothesis generating and large, prospective trials designed to assess for cancer recurrence and long-term survival are necessary to confirm these results.

\section{Declarations}

Conflicts of interest: Michael Grant receives salary support from the Agency for Healthcare Research and Quality (AHRQ; HHSP233201500020I) and serves on the Execute Board of the ERAS Cardiac Society. Gabriel Mena has an academic grant from Pacira Pharmaceuticals. Javier Ripolles-Melchor receives honoraria as a consultant for Edwards Lifesciences and Fresenius Kabi. All other authors have no competing interests.

Funding/Support: This research did not receive any specific grant from funding agencies in the public, commercial, or non-for-profit sectors.

Correspondence: Andres Zorrilla-Vaca MD, Department of Anesthesiology and Perioperative Medicine, The University of Texas MD Anderson Cancer Center, Houston, TX. Email: andres.zorrilla@correounivalle.edu.co 


\section{References}

1. Platell CFE. Changing patterns of recurrence after treatment for colorectal cancer. Int $\mathrm{J}$ Colorectal Dis $2007 ; 22: 1223-31$.

2. Simard J, Kamath S, Kircher S. Survivorship Guidance for Patients with Colorectal Cancer. Curr Treat Options Oncol 2019;20:38.

3. Rieth EF, Fischer GW, Afonso AM. Organization of Multidisciplinary Cancer Care for the Surgical Patient: Role of Anesthesiologists. Curr Anesthesiol Rep 2018;8:368-74.

4. Wang X, Zhao J, Shen Z, Fairweather M, Enzinger PC, Sun Y, Wang J. Multidisciplinary Approach in Improving Survival Outcome of Early-Stage Gastric Cancer. J Surg Res 2020;255:285-96.

5. Wall T, Sherwin A, Ma D, Buggy DJ. Influence of perioperative anaesthetic and analgesic interventions on oncological outcomes: a narrative review. Br J Anaesth 2019;123:135-50.

6. Chagpar AB. Enhanced Recovery After Surgery: Moving Toward Best Practice. Ann Surg Oncol 2021. Available at: http://link.springer.com/10.1245/s10434-021-09846-7. Accessed April 10, 2021.

7. Gustafsson UO, Scott MJ, Hubner M, Nygren J, Demartines N, Francis N, Rockall TA, Young-Fadok TM, Hill AG, Soop M, Boer HD de, Urman RD, Chang GJ, Fichera A, Kessler H, Grass F, Whang EE, Fawcett WJ, Carli F, Lobo DN, Rollins KE, Balfour A, Baldini G, Riedel B, Ljungqvist O. Guidelines for Perioperative Care in Elective Colorectal Surgery: Enhanced Recovery After Surgery (ERAS $\AA$ ) Society Recommendations: 2018. World J Surg 2019;43:659-95.

8. Hamad A, Pawlik TM, Ejaz A. Guide to Enhanced Recovery for Cancer Patients Undergoing Surgery: Pancreaticoduodenectomy. Ann Surg Oncol 2021.

9. Slim K, Regimbeau JM. Increased survival might be an unexpected additional advantage of enhanced recovery after surgery programs. Journal of Visceral Surgery 2018;155:169-71.

10. Quiram BJ, Crippa J, Grass F, Lovely JK, Behm KT, Colibaseanu DT, Merchea A, Kelley SR, Harmsen WS, Larson DW. Impact of enhanced recovery on oncological outcomes following minimally invasive surgery for rectal cancer. British Journal of Surgery 2019;106:922-9.

11. Viannay P, Hamy A, Jaouen R, Caroli-Bosc FX, Luel C, Vasseur S, Levaillant M, Hamel JF, Venara A. Does enhanced recovery improve the survival rates of patients 3 years after undergoing surgery to remove a tumor in the colon? Int J Colorectal Dis 2019;34:441-9.

12. Gustafsson UO, Oppelstrup H, Thorell A, Nygren J, Ljungqvist O. Adherence to the ERAS protocol is Associated with 5-Year Survival After Colorectal Cancer Surgery: A Retrospective Cohort Study. World J Surg 2016;40:1741-7. 
13. Lohsiriwat V, Lertbannaphong S, Polakla B, Riansuwan W. Implementation of enhanced recovery after surgery and its increasing compliance improved 5-year overall survival in resectable stage III colorectal cancer. Updates Surg 2021. Available at: http://link.springer.com/10.1007/s13304-021-010048. Accessed April 10, 2021.

14. Compton C, Fenoglio-Preiser CM, Pettigrew N, Fielding LP. American Joint Committee on Cancer Prognostic Factors Consensus Conference: Colorectal Working Group. Cancer 2000;88:1739-57.

15. Trépanier M, Minnella EM, Paradis T, Awasthi R, Kaneva P, Schwartzman K, Carli F, Fried GM, Feldman LS, Lee L. Improved Disease-free Survival After Prehabilitation for Colorectal Cancer Surgery. Ann Surg 2019;270:493-501.

16. Curtis NJ, Taylor M, Fraser L, Salib E, Noble E, Hipkiss R, Allison AS, Dalton R, Ockrim JB, Francis NK. Can the combination of laparoscopy and enhanced recovery improve long-term survival after elective colorectal cancer surgery? Int J Colorectal Dis 2018;33:231-4.

17. Asklid D, Segelman J, Gedda C, Hjern F, Pekkari K, Gustafsson UO. The impact of perioperative fluid therapy on short-term outcomes and 5-year survival among patients undergoing colorectal cancer surgery - A prospective cohort study within an ERAS protocol. Eur J Surg Oncol 2017;43:1433-9.

18. Heaney A, Buggy DJ. Can anaesthetic and analgesic techniques affect cancer recurrence or metastasis? Br J Anaesth 2012;109 Suppl 1:i17-28.

19. Holler JPN, Ahlbrandt J, Burkhardt E, Gruss M, Röhrig R, Knapheide J, Hecker A, Padberg W, Weigand MA. Peridural analgesia may affect long-term survival in patients with colorectal cancer after surgery (PACO-RAS-Study): an analysis of a cancer registry. Ann Surg 2013;258:989-93.

20. Day A, Smith R, Jourdan I, Fawcett W, Scott M, Rockall T. Retrospective analysis of the effect of postoperative analgesia on survival in patients after laparoscopic resection of colorectal cancer. $\mathrm{Br} \mathrm{J}$ Anaesth 2012;109:185-90.

21. Ripollés-Melchor J, Fuenmayor-Varela ML de, Camargo SC, Fernández PJ, Barrio ÁCD, MartínezHurtado E, Casans-Francés R, Abad-Gurumeta A, Ramírez-Rodríguez JM, Calvo-Vecino JM. [Enhanced recovery after surgery protocol versus conventional perioperative care in colorectal surgery. A single center cohort study]. Rev Bras Anestesiol 2018;68:358-68.

22. Watt DG, McSorley ST, Horgan PG, McMillan DC. Enhanced Recovery After Surgery: Which Components, If Any, Impact on The Systemic Inflammatory Response Following Colorectal Surgery?: A Systematic Review. Medicine (Baltimore) 2015;94:e1286.

23. Cabellos Olivares M, Labalde Martínez M, Torralba M, Rodríguez Fraile JR, Atance Martínez JC. Creactive protein as a marker of the surgical stress reduction within an ERAS protocol (Enhanced Recovery After Surgery) in colorectal surgery: A prospective cohort study. J Surg Oncol 2018;117:717-24. 
24. Venara A, Duchalais E, Dariel A, Aubert P, Durand T, Meurette G, Rolli-Derkinderen M, Hamy A, Neunlist M. Anti-inflammatory Effects of Enhanced Recovery Programs on Early-Stage Colorectal Cancer Surgery. World J Surg 2018;42:953-64.

25. Jaloun HE, Lee IK, Kim MK, Sung NY, Turkistani SAA, Park SM, Won DY, Hong SH, Kye B-H, Lee YS, Jeon HM. Influence of the Enhanced Recovery After Surgery Protocol on Postoperative Inflammation and Short-term Postoperative Surgical Outcomes After Colorectal Cancer Surgery. Ann Coloproctol 2020;36:264-72.

\section{Tables}

Table 1. Patient demographics and clinical characteristics before and after institution of an ERAS program for colorectal surgery 


\begin{tabular}{|c|c|c|c|c|}
\hline Variables & $\begin{array}{l}\text { Overall } \\
(n=646)\end{array}$ & $\begin{array}{l}\text { Pre-ERAS } \\
(n=339)\end{array}$ & $\begin{array}{l}\text { ERAS } \\
(n=307)\end{array}$ & $P$ value \\
\hline Age & 73 [63-80] & 72 [62-79] & 74 [64-80] & 0.15 \\
\hline Female & $233(36.4 \%)$ & $109(32.5 \%)$ & $124(40.7 \%)$ & 0.03 \\
\hline \multicolumn{5}{|l|}{$\mathrm{BMI}, \mathrm{kg} \cdot \mathrm{m}^{-2}$} \\
\hline$\leq 18.5$ & $3(0.5 \%)$ & $2(0.9 \%)$ & $1(0.5 \%)$ & 0.24 \\
\hline $18.5-25$ & $108(16.7 \%)$ & 47 (13.9\%) & $61(19.9 \%)$ & \\
\hline $25-30$ & $217(33.6 \%)$ & $119(35.1 \%)$ & $98(31.9 \%)$ & \\
\hline$\geq 30$ & $123(19.0 \%)$ & $60(17.7 \%)$ & $63(20.5 \%)$ & \\
\hline Missing & $195(30.2 \%)$ & $111(32.7 \%)$ & $84(27.4 \%)$ & \\
\hline ASA & & & & 0.08 \\
\hline I & $53(8.3 \%)$ & $32(9.6 \%)$ & $21(6.9 \%)$ & \\
\hline II & $360(56.3 \%)$ & 189 (56.4\%) & 171 (56.1\%) & \\
\hline III & $217(33.9 \%)$ & $112(33.4 \%)$ & $105(34.4 \%)$ & \\
\hline IV & $9(1.4 \%)$ & $1(0.3 \%)$ & $8(2.6 \%)$ & \\
\hline Anemia & $302(46.7 \%)$ & 159 (46.9\%) & $143(46.6 \%)$ & 0.94 \\
\hline Albumin & 4 [3.5-4.3] & 4 [3.5-4.3] & 4 [3.4-4.3] & 0.11 \\
\hline Hypertension & $383(59.8 \%)$ & $192(57.3 \%)$ & $191(62.6 \%)$ & 0.17 \\
\hline Diabetes & $163(25.5 \%)$ & $86(25.7 \%)$ & 77 (25.3\%) & 0.90 \\
\hline COPD & $96(15.0 \%)$ & $55(16.4 \%)$ & $41(13.4 \%)$ & 0.29 \\
\hline Kidney disease & $58(9.1 \%)$ & $34(10.2 \%)$ & $24(7.9 \%)$ & 0.32 \\
\hline Cirrhosis & $61(9.5 \%)$ & $37(11.0 \%)$ & $24(7.9 \%)$ & 0.17 \\
\hline Surgery time & 125 [99-167] & 140 [110-180] & 119 [90-145] & $<0.01$ \\
\hline Epidural & $101(15.8 \%)$ & $70(21.0 \%)$ & $31(10.2 \%)$ & $<0.01$ \\
\hline Anesthesia & & & & $<0.01$ \\
\hline Inhaled & $514(80.9 \%)$ & $285(86.1 \%)$ & $229(75.3 \%)$ & \\
\hline Intravenous & $121(19.1 \%)$ & 46 (13.9\%) & $75(24.7 \%)$ & \\
\hline Fluid balance & 2351 [1302-3393] & 2783 [1687-3658] & 1970 [1049-2873] & $<0.01$ \\
\hline Conversion & $31(4.8 \%)$ & $16(4.8 \%)$ & $15(4.9 \%)$ & 0.93 \\
\hline
\end{tabular}




\begin{tabular}{lllll} 
Stoma & $122(19.1 \%)$ & $67(20.0 \%)$ & $55(18.0 \%)$ & 0.53 \\
\hline Cancer stage & & & & 0.14 \\
\hline In-situ & $114(18.5 \%)$ & $68(20.9 \%)$ & $46(15.8 \%)$ & \\
\hline I & $114(18.5 \%)$ & $55(16.9 \%)$ & $59(20.2 \%)$ & \\
\hline II & $154(25.0 \%)$ & $80(24.7 \%)$ & $74(25.3 \%)$ & \\
\hline III & $160(25.9 \%)$ & $76(23.5 \%)$ & $84(28.8 \%)$ & \\
\hline IV & $74(12.0 \%)$ & $45(13.9 \%)$ & $29(9.9 \%)$ &
\end{tabular}

ASA: American Society of Anesthesiologists, COPD: chronic obstructive pulmonary disease.

Table 2. Univariate analysis of clinical variables for oncologic recurrence and 5-year survival 


\begin{tabular}{|c|c|c|c|}
\hline \multirow[t]{3}{*}{ Variable } & \multirow{3}{*}{$\begin{array}{l}\text { Disease-Free Survivors } \\
(n=239)\end{array}$} & \multicolumn{2}{|c|}{ Affected Patients } \\
\hline & & Recurrence & Deaths \\
\hline & & $(n=123)$ & $(n=176)$ \\
\hline Age & $71[61-79]$ * & $74[63-79]+$ & $77[70-82] \neq$ \\
\hline Female & $64(27.6 \%)$ & $33(26.8 \%)$ & $47(27.8 \%)$ \\
\hline \multicolumn{4}{|l|}{$\mathrm{BMI}, \mathrm{kg} \cdot \mathrm{m}^{-2}$} \\
\hline$\leq 18.5$ & $0(0 \%)$ & $0(0 \%)$ & $0(0 \%)$ \\
\hline $18.5-25$ & $46(12 \%)$ & $20(16.2 \%)$ & 35 (19.9\%) \\
\hline $25-30$ & $76(31.8 \%)$ & 41 (33.3\%) & $60(34.1 \%)$ \\
\hline$\geq 30$ & $39(16.3 \%)$ & $17(13.8 \%)$ & $28(15.9 \%)$ \\
\hline Missing & 78 (32.6\%) & $45(36.6 \%)$ & $53(30.1 \%)$ \\
\hline \multicolumn{4}{|l|}{ ASA } \\
\hline 1 & $17(7.1 \%)$ & $11(8.9 \%)$ & $7(3.9 \%)$ \\
\hline II & $104(43.5 \%)$ & $64(52.0 \%)$ & $72(40.9 \%)$ \\
\hline III & $106(44.4 \%)$ * & $47(38.2 \%)$ & $85(48.3 \%) \ddagger$ \\
\hline IV & $5(2.1 \%)$ & $1(0.8 \%)$ & $5(2.8 \%) \ddagger$ \\
\hline Missing & $7(2.9 \%)$ & $0(0 \%)$ & $7(3.9 \%)$ \\
\hline Anemia & $134(56.1 \%)$ & $63(51.2 \%)$ & $106(60.2 \%)$ \\
\hline Albumin & $3.8[3.2-4.2]$ & $4[3.6-4.2]$ & $3.7[3.1-4.2]$ \\
\hline Hypertension & $149(64.2 \%)$ & $76(61.8 \%)$ & $111(65.7 \%)$ \\
\hline Diabetes mellitus & $70(30.2 \%)$ * & $41(33.3 \%) \dagger$ & $52(30.8 \%)$ \\
\hline COPD & $48(20.7 \%)$ * & $18(14.6 \%)$ & $37(21.9 \%) \ddagger$ \\
\hline CKD & $28(12.1 \%)$ * & $10(8.1 \%)$ & $24(14.2 \%) \neq$ \\
\hline Cirrhosis & $24(10.3 \%)$ & $10(8.1 \%)$ & $17(10.1 \%)$ \\
\hline Surgery time & 130 [104-179] * & 131 [106-170] & $131[106-179] \ddagger$ \\
\hline Epidural & $43(18.5 \%)$ & $20(16.3 \%)$ & $32(18.9 \%)$ \\
\hline \multicolumn{4}{|l|}{ Anesthesia } \\
\hline Inhaled & $189(82.3 \%)$ & $104(85.3 \%)$ & $136(80.9 \%)$ \\
\hline Intravenous & 41 (17.8\%) & 18 (14.8\%) & 32 (19.1\%) \\
\hline
\end{tabular}




\begin{tabular}{|llll|}
\hline Fluid balance & $2611[1690-3590] *$ & $2648[1761-3528]$ & $2844[1693-3885] \ddagger$ \\
\hline Conversion & $12(5.2 \%)$ & $8(6.5 \%)$ & $8(4.7 \%)$ \\
\hline Stoma & $59(25.4 \%) *$ & $29(23.6 \%)$ & $44(26.0 \%) \ddagger$ \\
\hline Cancer stage & & & \\
\hline In-situ & $23(9.6 \%) *$ & $8(6.5 \%) \dagger$ & $18(10.2 \%) \ddagger$ \\
\hline I & $24(10.0 \%) *$ & $9(7.3 \%) \dagger$ & $22(12.5 \%) \ddagger$ \\
\hline III & $54(22.6 \%)$ & $36(29.3 \%)$ & $33(18.8 \%)$ \\
\hline IV & $71(29.7 \%) *$ & $45(36.6 \%) \dagger$ & $48(27.3 \%) \ddagger$ \\
\hline Unknown & $53(22.2 \%) *$ & $22(17.9 \%) \dagger$ & $43(24.4 \%) \ddagger$ \\
\hline
\end{tabular}

ASA: American Society of Anesthesiologists, COPD: chronic obstructive pulmonary disease, CKD: chronic kidney disease.

${ }^{*} \mathrm{P}<0.05$ comparing with patients who died or recurred.

$+\mathrm{P}<0.05$ comparing with patients who recurred.

$\ddagger \mathrm{P}<0.05$ comparing with patients who survived.

\section{Figures}




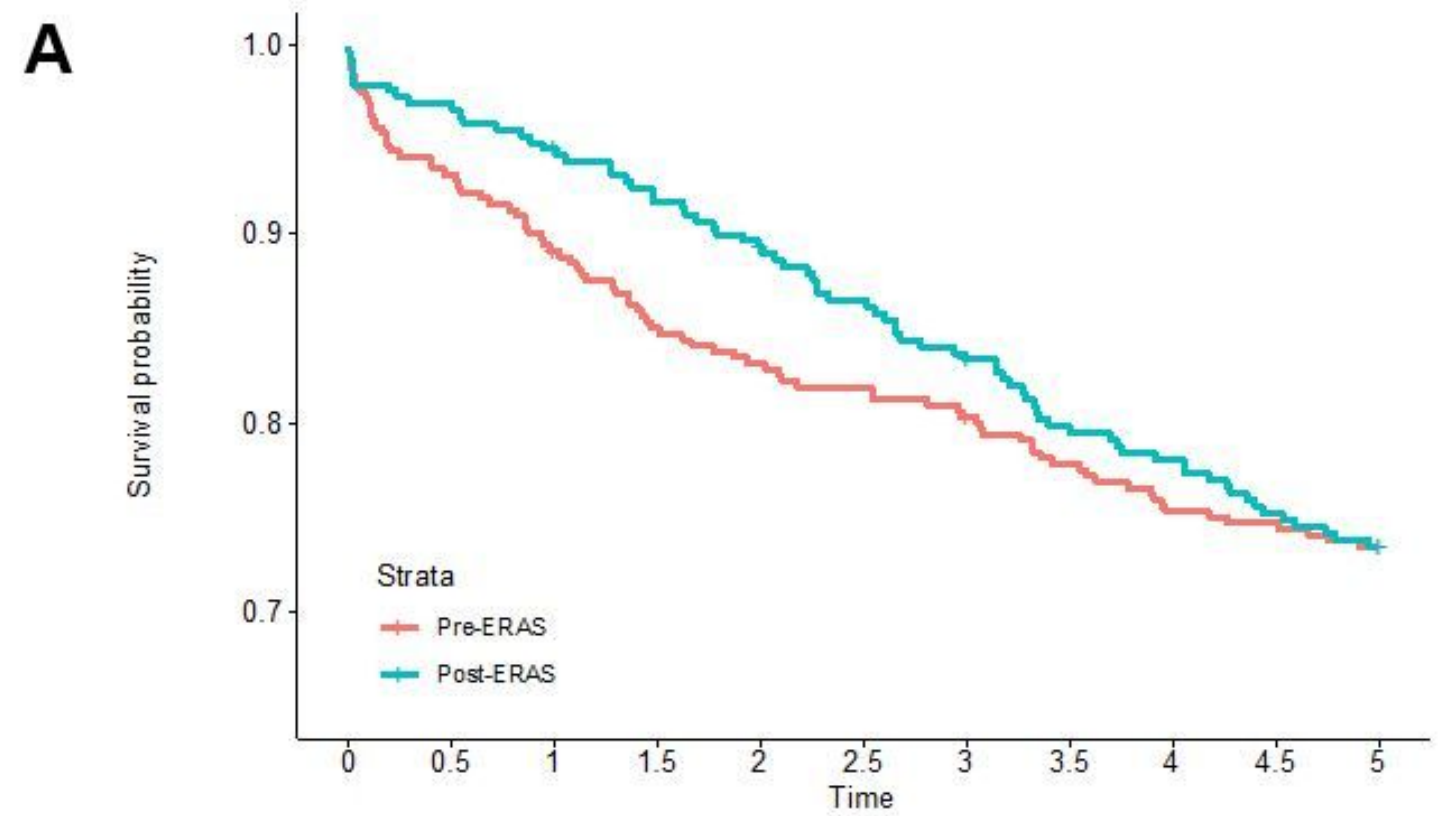

Number at risk
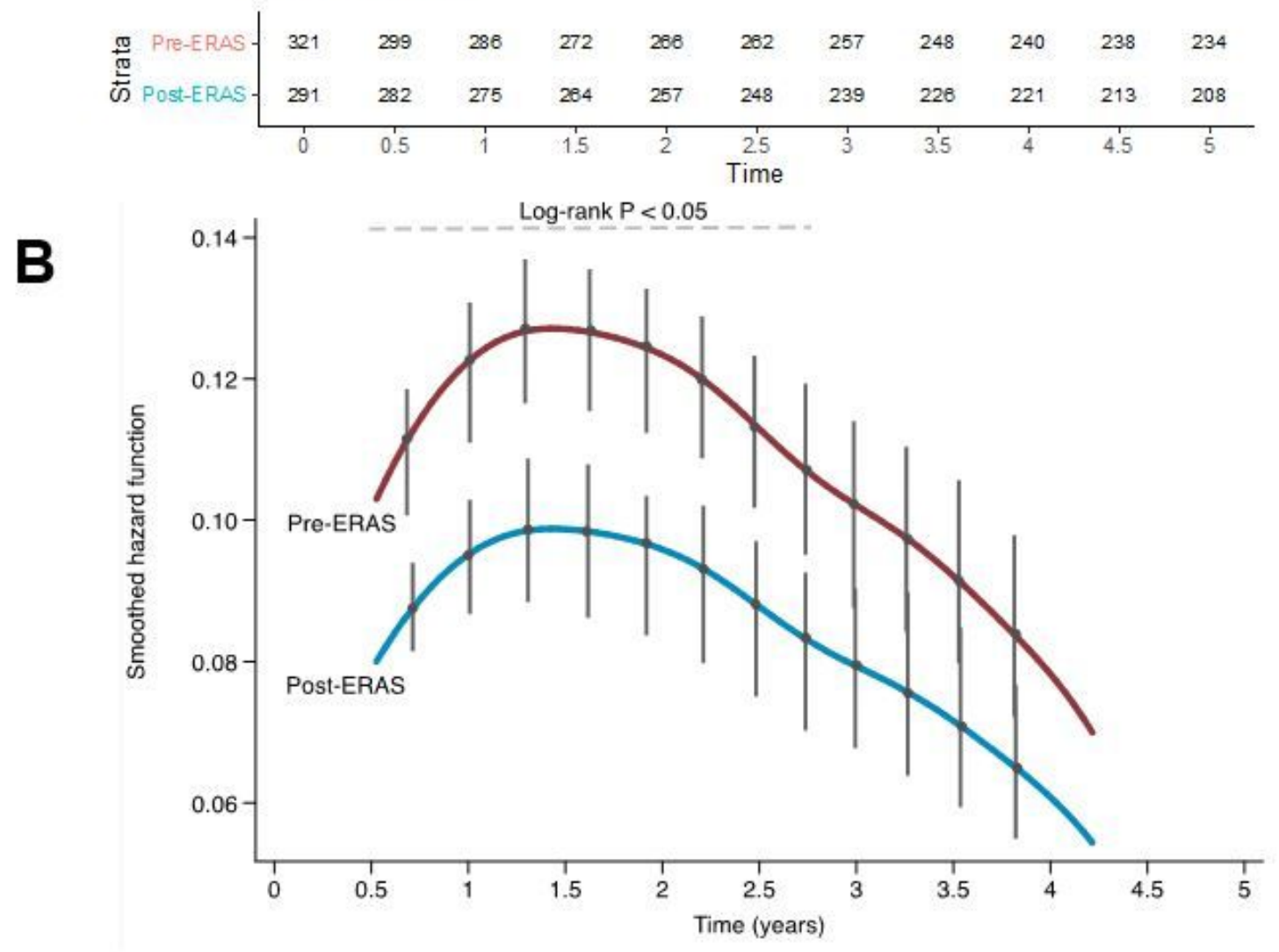

\section{Figure 1}

Survival analysis comparing pre-ERAS and ERAS. (A) Kaplan-Meier curves of each period, (B) Cox hazard function of mortality rates for each period. 

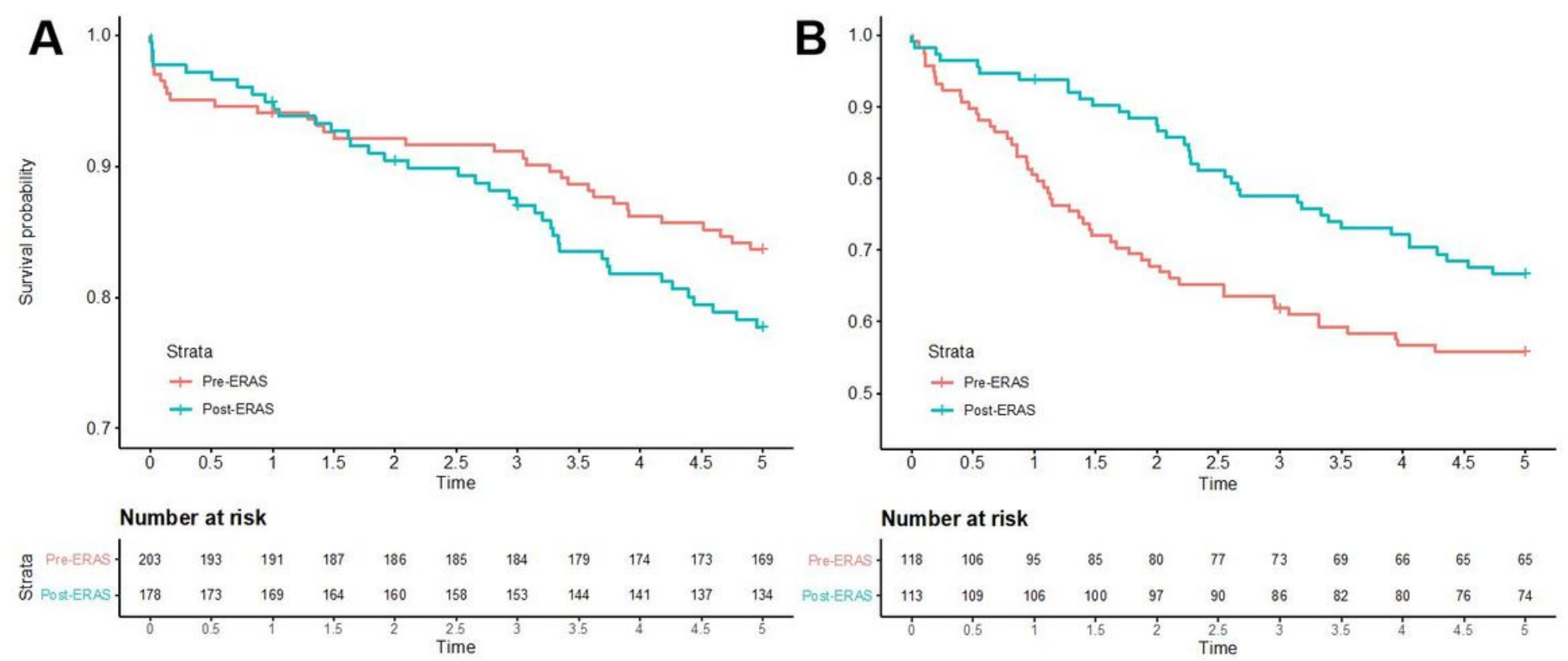

\section{Figure 2}

Subgroup analysis showing survival rates stratified based upon low cancer stage (panel A) and advanced cancer stage (panel B). 